\title{
Autoeficácia docente e Educação Especial: Revisão da produção de conhecimento nacional e internacional com ênfase na formação de professores
}

Teacher self-efficacy and Special Education: national and international knowledge production review with emphasis in teacher education

Autoeficacia docente y Educación Especial: Revisión de la producción de conocimiento nacional e internacional con énfasis en la formación de profesores

\author{
* Bárbara Amaral Martins \\ Professora Adjunta da Universidade Federal do Mato Grosso do Sul, Campus do \\ Pantanal.barbara.amts@gmail.com \\ ** Miguel Claudio Moriel Chacon \\ Professor doutor na Universidade Estadual Paulista, Marília, São Paulo, Brasil. \\ miguelchacon@marilia.unesp.br
}

Recebido em 03 de dezembro de 2018

Aprovado em 05 de junho de 2019

Publicado em 03 de julho de 2019

\section{RESUMO}

O professor tem papel fundamental na efetivação da educação inclusiva, sendo imprescindíveis, conhecimentos, motivação e recursos. No âmbito motivacional, ressaltase a importância da autoeficácia docente, compreendida como o julgamento do professor a respeito de suas capacidades profissionais. Se um professor tem dúvidas sobre suas competências didáticas, tende a visualizar um cenário de insucesso que influi negativamente sobre seu esforço e dedicação, evidenciando a importância de altos níveis de autoeficácia, em especial, no trabalho com estudantes público da Educação Especial. Logo, é relevante buscar estratégias que a fortaleçam, de maneira que a formação docente pode ser uma alternativa. O objetivo deste estudo foi verificar a produção de conhecimentos sobre autoeficácia docente no contexto da Educação Especial no Brasil e no exterior, e, analisar as pesquisas que investigaram os efeitos da formação sobre a autoeficácia de professores para a inclusão escolar de estudantes com deficiência, transtornos globais do desenvolvimento ou altas habilidades/superdotação. Revisaram-se, sistematicamente, artigos, teses e dissertações levantados a partir de seis bases de dados nacionais e internacionais, totalizando 74 produções que relacionam autoeficácia docente e Educação Especial, cinco delas, brasileiras. A análise ocorreu a partir da leitura dos trabalhos, elaboração de quadros e estatística descritiva. Do total, as 12 pesquisas que envolviam formação de professores foram analisadas qualitativamente e demonstraram que a 
formação pode aumentar os níveis de autoeficácia docente, sendo que tal probabilidade é ampliada com a articulação entre teoria e prática bem-sucedida. Enfatiza-se a potencialidade das experiências vicárias na formação docente enquanto uma das principais fontes de autoeficácia.

Palavras-chave: Autoeficácia docente; Formação docente; Educação Especial.

\section{ABSTRACT}

The teacher has a fundamental role in the inclusive education realization, being essential, knowledge, motivation and resources. In the motivational context, the importance of teacher self-efficacy, understood as the teacher's judgment about his professional capacities, is emphasized. If a teacher has doubts about his/her teaching abilities, he/she tends to visualize a failure scenario that negatively influences his/her effort and dedication, evidencing the importance of high self-efficacy levels, especially in the work with students Special Education public. Therefore, it is important to seek strategies that strengthen it, so that teacher education can be an alternative. This study objective is to verify the knowledge production about teacher self-efficacy in the Special Education context in Brazil and abroad, and to analyze the researches that investigated the education effects on teachers' selfefficacy for school inclusion of students with disabilities, pervasive developmental disorders or giftedness. Articles, theses and dissertations collected from six national and international databases were systematically reviewed, totaling 74 works that relate to self-efficacy and Special Education, five of which are Brazilian. The analysis was based on the reading of the works, elaboration of tables and descriptive statistics. Of the total, 12 researches involving teacher education were qualitatively analyzed and demonstrated that education can increase teacher self-efficacy levels, so that this probability is enhanced by the link between theory and successful practice. It emphasizes the vicarious experiences potentiality in teacher education as one of the main self-efficacy sources.

Keywords: Teacher self-efficacy; Teacher education; Special Education.

\section{RESUMEN}

El profesor tiene un papel fundamental en la efectividad de la educación inclusiva, siendo imprescindibles, conocimientos, motivación y recursos. En el ámbito motivacional, se resalta la importancia de la autoeficacia docente, comprendida como el juicio del profesor acerca de sus capacidades profesionales. Si un profesor tiene dudas sobre sus competencias didácticas, tiende a visualizar un escenario de fracaso que influye negativamente sobre su esfuerzo y dedicación, evidenciando la importancia de altos niveles de autoeficacia, en especial, en el trabajo con estudiantes público de la Educación Especial. Por lo tanto, es relevante buscar estrategias que la fortalezcan, de manera que la formación docente puede ser una alternativa. El objetivo de este estudio es verificar la producción de conocimientos acerca de la autoeficacia docente en el contexto de la Educación Especial en Brasil y en el exterior y analizar las investigaciones que investigaron los efectos de la formación sobre la autoeficacia de profesores para la inclusión escolar de alumnos con discapacidad, trastornos globales del desarrollo o superdotación. Se revisaron sistemáticamente artículos, tesis y disertaciones levantadas a partir de seis bases de datos 
nacionales e internacionales, totalizando 74 producciones que relacionan autoeficacia docente y Educación Especial, cinco de ellas, brasileñas. El análisis ocurrió a partir de la lectura de los trabajos, elaboración de cuadros y estadística descriptiva. Del total, las 12 investigaciones que involucraba formación de profesores se analizaron cualitativamente y demostraron que la formación puede aumentar los niveles de autoeficacia docente, siendoque tal probabilidad se amplía con la articulación entre teoría y práctica exitosa. Enfatiza la potencialidad de las experiencias vicarias en la formación docente como una de las principales fuentes de autoeficacia.

Palabras clave: Autoeficacia docente; Formación docente; Educación Especial.

\section{Introdução}

A educação inclusiva é um caminho sem volta, fruto de um compromisso globalmente assumido, rumo a uma sociedade mais justa e humana. A concretização desse projeto exige conhecimentos, recursos e disponibilidade por parte de todos. Nesse sentido, destacamos a importância da figura do professor, profissional a quem compete conduzir o processo de ensino e aprendizagem de maneira que todos os estudantes possam participar e aprender, num ambiente onde predomina o clima de respeito e solidariedade. No entanto, há situações em que o professor se vê temeroso diante da tarefa de ensinar estudantes que se afastam do perfil tipicamente idealizado.

Embora a educação inclusiva destine-se a promover a participação e a aprendizagem de todo e qualquer estudante, destacamos, especificamente, a inclusão do público da Educação Especial ${ }^{1}$, isto é, aqueles que apresentam deficiência, transtornos globais do desenvolvimento ou altas habilidades/superdotação (BRASIL, 1996). Quando o professor não acredita que possui as competências necessárias para atuar junto aos estudantes público da Educação Especial, pode apresentar barreiras atitudinais impeditivas para o trabalho colaborativo com o professor especialista, bem como atribuir pouca funcionalidade aos recursos materiais disponíveis, além do sentimento de frustração que pode desenvolver.

De acordo com Bandura (1989), a postura de iniciar, persistir ou abandonar uma ação é afetada pelas crenças do indivíduo sobre suas competências e capacidades para

\footnotetext{
${ }^{1}$ A Educação Especial é uma área de conhecimento e também uma modalidade de educação escolar que contempla os estudantes com deficiência, transtornos globais do desenvolvimento e altas habilidades/superdotação. Enquanto modalidade educacional, e na perspectiva da educação inclusiva, perpassa todos os níveis de ensino e ocorre, preferencialmente, nas instituições de ensino regular. Com vistas a atender às necessidades educacionais de seu público, envolve elaboração e disponibilização de recursos materiais, adaptações curriculares, uso de comunicação alternativa, itinerância, ensino colaborativo, além de Atendimento Educacional Especializado, entre outros.
} 
enfrentar as exigências do ambiente, ou seja, por sua autoeficácia. Quando a autoeficácia para realização de determinada ação está elevada, a pessoa visualiza um cenário de sucesso que se reflete positivamente na quantidade de esforço e no tempo empregado para superar obstáculos e atingir objetivos. Ao passo que quando se percebe ineficaz, o indivíduo torna-se propenso a visualizar cenários de insucesso que influenciam negativamente sua atuação. Posto isso, compreende-se que a autoeficácia docente é um constructo de extrema relevância para a inclusão escolar dos estudantes com deficiência, transtornos globais do desenvolvimento ou altas habilidades/superdotação.

Em razão da imprescindibilidade das capacidades docentes, ressalta-se a importância da formação continuada de professores (AZZI; POLYDORO; BZUNECK, 2006; MARTíNEZ, 2004). Essa formação deve procurar abarcar mais do que conhecimentos e habilidades, voltando-se também, para o desenvolvimento da autoeficácia, a qual influi sobre o aproveitamento do processo formativo, e, além disso, "o verdadeiramente importante não é possuir certas capacidades, mas sentir-se capaz de utilizá-las adequadamente" (MARTÍNEZ, 2004, p. 181, tradução nossa). Isso significa que a posse da capacidade é necessária, porém não suficiente, pois, com baixa autoeficácia, o indivíduo não encontra motivação para exercer a sua capacidade, uma vez que imagina resultados negativos dessa ação. Nesse contexto, Martínez (2004) esclarece que a motivação está intrinsicamente relacionada com a autoeficácia, as expectativas de resultado e as metas estabelecidas, fatores intimamente ligados às ações. Destarte, uma autoeficácia elevada favorece a busca e a persistência na aquisição de outras habilidades necessárias para a superação de novos desafios.

Feitas as considerações iniciais sobre a relevância da autoeficácia docente, elege-se este constructo como foco da presente pesquisa. A pesquisa científica tem como finalidade a busca de respostas para problemas ainda não solucionados, ou, ao menos, a indicação de possíveis caminhos para a sua resolução. Dessa maneira, deve ser constantemente avaliada para que cumpra o seu papel de atender aos interesses e necessidades da sociedade (SILVA; GAMBOA, 2011).

Sob esse prisma, faz-se necessário conhecer as produções de uma determinada área para verificar aquilo que já está suficientemente investigado e o que ainda requer novos esforços. 
Segundo Omote (2014) durante a produção de conhecimento, o pesquisador atua como um intermediário entre a realidade e a informação obtida, a partir da apreensão do objeto investigado. Essa apreensão não pode estar desassociada dos conhecimentos anteriormente produzidos. Em razão da importância desses conhecimentos para o desenvolvimento de novas pesquisas, temos por objetivo verificar a produção de conhecimentos científicos acerca da autoeficácia docente no contexto da Educação Especial no Brasil e no exterior e analisar as pesquisas que investigaram os efeitos da formação sobre a autoeficácia de professores para a inclusão escolar de estudantes com deficiência, transtornos globais do desenvolvimento e altas habilidades/superdotação.

\section{Método}

Iniciamos o estudo com uma revisão sistemática de teses e dissertações defendidas no Brasil com o objetivo de sistematizar os conhecimentos produzidos em território nacional sobre autoeficácia docente. Tal procedimento utilizou para consulta o Banco de Teses e Dissertações da CAPES e a Biblioteca Digital Brasileira de Teses e Dissertações (BDTD), tendo-se como descritores: autoeficácia, auto-eficácia ou eficácia, associados aos termos professor ou docente.

Para o levantamento das teses e dissertações nacionais sobre autoeficácia docente, tivemos como critérios de inclusão e exclusão a seleção de publicações realizadas até o ano de 2017, cuja autoeficácia docente consistisse em objeto de estudo do trabalho, o que foi verificado a partir da leitura dos títulos e resumos, eliminando-se as duplicações. Já para o levantamento global de pesquisas no contexto da Educação Especial, os trabalhos deveriam ter como objeto de estudo a autoeficácia docente em associação à educação inclusiva e terem sido publicados até o ano de 2017. Como a educação inclusiva não se restringe ao público da Educação Especial, a leitura dos títulos e resumos foi o procedimento adotado para a seleção dos trabalhos. Em relação aos critérios de exclusão, eliminamos as duplicações de pesquisas entre as diferentes bases de dados e as produções que se referiam a outros estudantes além dos pertencentes ao público da Educação Especial, segundo a legislação brasileira: pessoas com deficiência, transtornos globais do desenvolvimento e altas habilidades/superdotação.

Em continuidade, realizamos nova revisão com o propósito de conhecer, globalmente, as pesquisas que relacionam autoeficácia docente e Educação Especial na perspectiva da 
educação inclusiva. Para isso, elegemos duas bases de dados nacionais (Scientific Electronic Library Online - Scielo e Portal de Periódicos CAPES) e duas, internacionais (Education Resources InformationCenter - ERIC e ScienceDirect). Salienta-se que o Portal de Periódicos CAPES foi usado tanto para busca de trabalhos nacionais quanto internacionais.

Para a realização da busca de produções internacionais, foram selecionados os descritores: teacher self-efficacy; special needs; inclusion; inclusive; disability; giftedness; gifted. Os descritores foram associados aos operadores booleanos AND e OR em adição ao uso da truncatura a partir do símbolo asterisco. A consulta foi realizada da seguinte maneira: self-efficacy AND teacher AND (special AND needs OR inclusiv* OR disabilit ${ }^{\star}$ OR gift $\left.^{*}\right)$.

A análise dos dados ocorreu a partir da leitura global dos trabalhos e elaboração de quadros de registro, onde eram transcritas informações sobre os estudos: autores e títulos, universidades, países de realização dos estudos, ano de publicação, nível e programa de pós-graduação (quando tese ou dissertação), objeto de estudo, objetivos, métodos e resultados. Os quadros foram analisados quantitativamente por meio de estatística descritiva e, em seguida, os resultados dos trabalhos que envolviam formação de professores foram qualitativamente analisados e relacionados.

\section{Resultados e Discussão}

Serão apresentados, separadamente, os resultados referentes às teses $\mathrm{e}$ dissertações nacionais sobre autoeficácia docente, às produções científicas sobre autoeficácia docente em relação à Educação Especial e, os estudos que focalizam a formação de professores para o fortalecimento das crenças de autoeficácia na perspectiva da educação inclusiva.

\section{A produção de conhecimento sobre autoeficácia docente nas teses e dissertações brasileiras}

Foram encontrados 34 trabalhos, dos quais nove teses de doutorado e 25 dissertações de mestrado. Essas pesquisas sobre autoeficácia docente dividem-se entre 12 universidades. A universidade com o maior número de produções nesta temática é a Universidade Estadual de Campinas (UNICAMP), com oito trabalhos. A Universidade Federal do Rio Grande do Sul (UFRGS) apresenta cinco teses e dissertações, seguida da 
Universidade Estadual de Londrina (UEL) e da Universidade do Oeste Paulista (UNOESTE), que apresentam quatro produções cada; na sequência, tem-se a Universidade Estadual Paulista "Júlio de Mesquita Filho" (UNESP) com três, juntamente com a Universidade Federal do Pará (UFPA). A Universidade Federal do Paraná (UFPR) apresenta duas produções, e por fim, com um trabalho estão: Universidade de São Paulo (USP), Universidade de Taubaté (UNITAU), Universidade Católica de Brasília (UCB), Universidade Católica de Pernambuco (UNICAP) e Universidade Federal da Bahia (UFBA). Observa-se que a maioria das pesquisas (17) foi desenvolvida em universidades do estado de São Paulo.

Das 34 teses e dissertações encontradas, 15 foram desenvolvidas em programas de pós-graduação em Educação (UNICAMP, UEL, UNESP e UNOESTE), sete, em programas de Música (UFRGS e UFPR) e, três, em programa de Teoria e pesquisa do comportamento (UFPA). Dois trabalhos foram realizados em programa de Educação Física (UNICAMP), sendo que cada um dos programas, Ciências da motricidade (UNESP), Psicologia (UCB), Psicologia clínica (UNICAP), Gestão e desenvolvimento regional (UNITAU), Ensino de ciências (USP), Ensino de ciências e educação matemática (UEL) e Administração (UFBA), apresentou uma tese ou dissertação. Entre os 15 programas de pós-graduação onde foram desenvolvidas as pesquisas sobre autoeficácia docente, nove receberam nota 4 na avaliação quadrienal 2017 da CAPES, seis recebem nota 5, e dois, nota 7 (CAPES, 2017). Os programas com nota 4 são considerados de bom desempenho; para os que apresentam apenas curso de mestrado, a nota máxima é 5; as notas 6 e 7 representam desempenho de alto padrão. Assim sendo, nota-se que esses estudos são provenientes de programas de qualidade. Vale salientar, ainda, que entre os quatro programas de pós-graduação em Educação, onde 13 pesquisas sobre autoeficácia docente foram desenvolvidas, três programas localizam-se no estado de São Paulo, onde se destaca a UNICAMP com seis dessas produções. A Figura 1 ilustra a distribuição das pesquisas de acordo com o ano em que foram publicadas.

Figura 1 - Distribuição das teses e dissertações segundo o ano de publicação

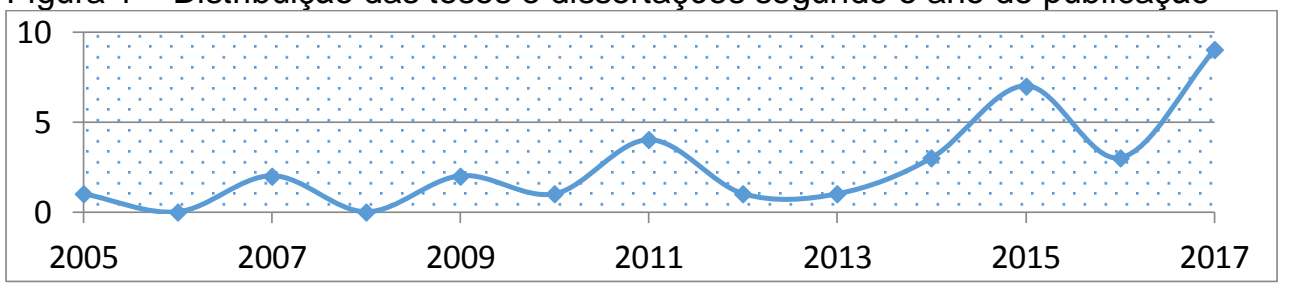

Fonte: Elaboração própria. 
Segundo o levantamento realizado, a primeira pesquisa brasileira sobre autoeficácia docente foi publicada no ano de 2005 e observa-se que até 2010 há uma oscilação entre zero e duas pesquisas anuais. Em 2011 ocorre uma elevação (4) que, no entanto, não se mantém nos anos seguintes. A partir de 2014, observa-se que o número de publicações assume certa constância, mostrando-se igual ou superior a três pesquisas, sendo que o maior número de publicações ocorreu em 2017 (9). Desse modo, nota-se que essa temática vem, gradativamente, despertando maior interesse por parte dos pesquisadores nacionais.

Essas investigações abordaram a autoeficácia docente sob diferentes perspectivas. A Tabela 1 apresenta a incidência das pesquisas conforme o objeto de estudo.

\begin{tabular}{lcc} 
Tabela 1 - Distribuição das teses e dissertações segundo o objeto de estudo investigado \\
\hline OBJETO DE ESTUDO & $\mathbf{f}$ & $\%$ \\
\hline Autoeficácia de professores de música & 7 & 20,59 \\
Autoeficácia de professores de educação física & 3 & 8,83 \\
Autoeficácia docente para o uso de tecnologias & 3 & 8,83 \\
Relação entre autoeficácia docente e burnout & 3 & 8,83 \\
Autoeficácia de professores iniciantes & 2 & 5,88 \\
Autoeficácia de professores universitários & 2 & 5,88 \\
Autoeficácia docente e educação física adaptada & 2 & 5,88 \\
Autoeficácia de professores do ensino médio & 1 & 2,94 \\
Relação entre autoeficácia docente e contexto de ensino & 1 & 2,94 \\
Relação entre autoeficácia docente e percepções de apoio & 1 & 2,94 \\
Relação entre autoeficácia docente e atribuição causal & 1 & 2,94 \\
Fontes de autoeficácia docente & 1 & 2,94 \\
Relação entre autoeficácia docente e bem-estar subjetivo & 1 & 2,94 \\
Autoeficácia do professor coordenador pedagógico & 1 & 2,94 \\
Autoeficácia coletiva e satisfação no trabalho & 1 & 2,94 \\
Relação entre autoeficácia docente, burnout e repercussões familiares & 1 & 2,94 \\
Autoeficácia para o ensino de arte & 1 & 2,94 \\
Autoeficácia docente e inclusão no Ensino Superior & 1 & 2,94 \\
Relação entre autoeficácia docente e otimismo acadêmico & 1 & 2,94 \\
\hline TOTAL & 34 & 100,00 \\
\hline
\end{tabular}

Fonte: Elaboração própria.

Com base na Tabela 1, verifica-se que há diversidade em relação ao objeto de estudo dessas pesquisas e constata-se que, no Brasil, a autoeficácia docente tem sido pouco investigada em relação à Educação Especial, sendo que entre os três estudos encontrados, dois se relacionam à educação física adaptada (VENDITTI JÚNIOR 2005; 2010) UNICAMP - e um, à inclusão de pessoas com deficiência no Ensino Superior (LEONARDO, 2017) - UNOESTE. 
Venditti Júnior (2005) investigou a autoeficácia de professores e futuros professores de educação física com atuação profissional ou formação acadêmica em educação física adaptada. Os 44 participantes responderam a uma escala de autoeficácia de professores de educação física e um questionário de atitude inclusiva, específica para professores de educação física. A análise dos dados indicou que a autoeficácia tem relação com a satisfação pessoal, com a disposição para prosseguir na carreira e com o esforço e a persistência empregados no desempenho da função docente. Verificou-se ainda, que a formação acadêmica, predominantemente teórica, pouco estimula o desenvolvimento das crenças de eficácia, o que poderia ser solucionado com a observação de profissionais mais experientes e vivências inclusivas diversas. $O$ autor ressalta a necessidade de um trabalho motivacional contínuo com esses profissionais, uma vez que os constantes desafios enfrentados no exercício da profissão podem prejudicar a autoeficácia.

Venditti Júnior (2010) analisou, ainda as crenças de autoeficácia de professores de educação física, a fim de aprofundar a investigação no contexto da educação física adaptada (EFA). Com uma amostra de 311 profissionais que atuavam ou já haviam atuado com EFA, foram respondidos os instrumentos: Escala de autoeficácia do professor de Educação Física, Escala Fontes de autoeficácia docente e Questionário de Atitude inclusiva do professor de Educação Física ou Questionário Associado sobre Aspectos Motivacionais. Os resultados reafirmaram a associação entre autoeficácia e fatores motivacionais: satisfação pessoal, disposição para continuar na carreira, níveis de esforço e persistência. Quanto às fontes de autoeficácia docente, as experiências diretas e as experiências vicárias se mostraram como as mais impactantes no contexto da EFA, seguidas dos aspectos fisiológicos e emocionais. Sendo assim, sugere a realização de Estágio Supervisionado e Práticas de Ensino envolvendo EFA durante a formação acadêmica, além do trabalho com as questões afetivas e emocionais no contexto de aprendizagem, bem como a participação em cursos e capacitações específicas e a observação de colegas e docentes atuando em EFA.

Leonardo (2017) teve por objetivo investigar as crenças de autoeficácia docente diante da inclusão de estudantes com necessidades educacionais especiais e os participantes da pesquisa foram nove professores de uma instituição privada de Ensino Superior. Esses professores ministravam disciplinas em turmas de oito cursos que tinham estudantes com deficiência matriculados. Os dados foram coletados por meio de grupo focal, com três 
encontros em que foram abordados os temas: Formação de professores e a escolha dessa profissão; Ações, desafios e expectativas em relação à inclusão; Crenças e fontes de autoeficácia. As discussões coletivas foram transcritas e submetidas à análise de conteúdo que revelou as categorias: Potencialidades e adversidades na inclusão, e, Autoeficácia e ações inclusivas. Na primeira, evidencia-se o despreparo dos docentes, os quais carecem de conhecimentos sobre estudantes com deficiência e estratégias de ensino adequadas, mas como potencialidades, buscam formação complementar e recursos pedagógicos que favoreçam a aprendizagem e o exercício da profissão por parte dos estudantes. $\mathrm{Na}$ segunda, a autoeficácia é associada às práticas inclusivas, pois os docentes revelam visualizar a capacidade de atingir resultados positivos e atuar com dedicação, criatividade e planejamento para que os mesmos se concretizem.

A seguir, a Tabela 2 exibe a distribuição das pesquisas segundo a natureza metodológica dos estudos.

Tabela 2 - Distribuição das pesquisas conforme a natureza metodológica dos estudos

\begin{tabular}{lcc}
\hline NATUREZA & $\mathbf{f}$ & $\%$ \\
\hline Quantitativa & 24 & 70,59 \\
Qualitativa & 4 & 11,76 \\
Quanti-qualitativa & 6 & 17,65 \\
\hline TOTAL & 34 & 100,00 \\
\hline
\end{tabular}

Fonte: Elaboração própria.

As teses e dissertações sobre autoeficácia docente envolveram, prioritariamente, métodos de pesquisa quantitativa, em especial, a aplicação de escalas e questionários. Seis estudos atrelaram os métodos quantitativos aos qualitativos, ao acrescentar entrevistas semiestruturadas, observações e/ou relatórios; ao passo que três pesquisas caracterizaram-se exclusivamente como qualitativas. Somente um dos estudos apresentou caráter interventivo, no qual Schmid (2015) analisou os efeitos de um curso de formação continuada sobre a autoeficácia de professores para o uso de mídias digitais em sala de aula, sem verificar alterações nos níveis de autoeficácia entre o pré-teste e o pós-teste.

A partir da análise das teses e dissertações brasileiras que tiveram a autoeficácia docente como objeto de estudo, verifica-se que esse constructo tem sido pouco explorado no que concerne à inclusão escolar do público da Educação Especial. 


\section{As pesquisas que relacionam autoeficácia docente e Educação Especial}

Quando a busca envolveu descritores em inglês, a base de dados ERIC apresentou 188 publicações, a ScienceDirect, 73, e o Portal de Periódicos CAPES, 29. Depois de aplicados os critérios de inclusão e exclusão, localizamos 67 produções. Quando os descritores foram empregados em idioma português, a base Scielo apresentou dois artigos e o Portal de Periódicos CAPES localizou três. Todos atendiam aos propósitos do estudo, apenas um estava duplicado e dois foram produzidos em Portugal.

Considerando também o levantamento de teses e dissertações nacionais, foram encontrados, ao todo, 74 trabalhos que tinham como objeto de estudo a autoeficácia docente em relação à Educação Especial na perspectiva da educação inclusiva. Desse total, 57 correspondem a artigos científicos publicados em periódicos (77,03\%). A Figura 2 revela o quantitativo anual desta produção, que vem ganhando maior expressividade desde 2010.

Figura 2 - Distribuição dos trabalhos segundo o ano de publicação

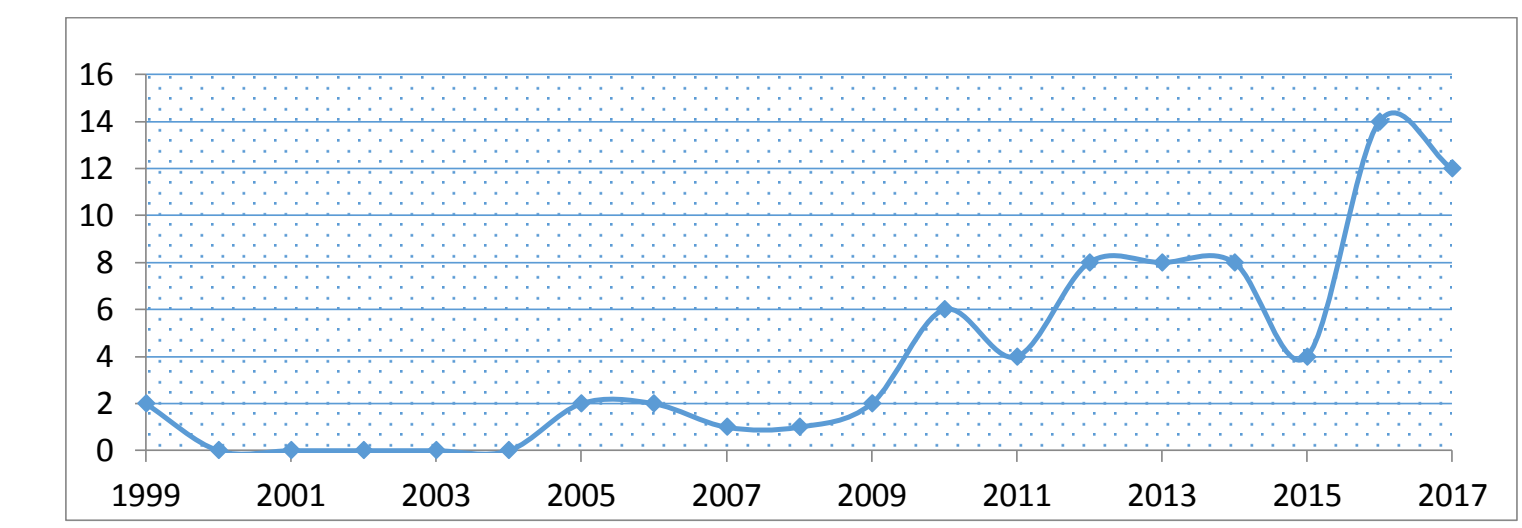

Fonte: Elaboração própria.

Observa-se que embora os primeiros estudos tenham sido publicados em 1999, não ocorrem outras publicações até 2005, ano em que passa a existir constância nesta produção, intensificada a partir de 2010.

Na sequência, o Quadro 1 sistematiza os países onde os estudos foram conduzidos. 
Quadro 1 - Distribuição dos países onde foram realizados estudos sobre autoeficácia docente e Educação Especial na perspectiva da educação inclusiva

\begin{tabular}{|l|c|l|c|}
\hline PAíS & $\mathbf{f}$ & PAíS & $\mathbf{f}$ \\
\hline Estados Unidos & 23 & Holanda & 2 \\
\hline China & 8 & Inglaterra & 1 \\
\hline Israel & 7 & Cingapura & 1 \\
\hline Austrália & 6 & Jamaica & 1 \\
\hline Brasil & 5 & México & 1 \\
\hline Canadá & 4 & Coréia & 1 \\
\hline Turquia & 4 & İndia & 1 \\
\hline Alemanha & 3 & Egito & 1 \\
\hline Portugal & 2 & Irlanda & 1 \\
\hline Paquistão & 2 & França & 1 \\
\hline Finlândia & 2 & Sérvia & 1 \\
\hline África do Sul & 2 & Áustria & 1 \\
\hline Japão & 2 & Escócia & \\
\hline
\end{tabular}

Fonte: Elaboração própria.

O Quadro 1 nos permite observar que, em relação à autoeficácia docente frente à inclusão do público da Educação Especial, a maioria das pesquisas investigou o contexto norte-americano, mais precisamente, os Estados Unidos, cuja produção é de 23 trabalhos envolvendo teses e artigos. A China, com oito artigos, foi o segundo país onde mais pesquisas foram desenvolvidas, seguida de Israel, com sete publicações em periódicos. O Brasil possui cinco publicações entre teses, dissertações e artigos científicos até o momento. Cabe destacar que alguns artigos abordavam mais de um país.

A baixa incidência de estudos brasileiros não é exclusividade dos que se relacionam com a Educação Especial, visto que laochite et al. (2016) realizaram um levantamento de artigos científicos sobre autoeficácia no contexto educacional, localizando 15 publicações de 2002 a 2013, as quais abrangiam: autoeficácia docente (seis), autoeficácia acadêmica (cinco); e autoeficácia no ensino superior (quatro). Os autores compreendem que as publicações nacionais acerca da autoeficácia na área educacional se encontram em "estado embrionário" (IAOCHITE et al. 2016, p. 48), mesmo porque parte dessas pesquisas se destina à validação de instrumentos construídos em outros países, além de haver uma concentração dessa produção nas regiões sudeste e sul do Brasil, principalmente, no estado de São Paulo.

Na sequência, a Tabela 3 distribui as publicações segundo a natureza dos estudos levantados. 
http://dx.doi.org/10.5902/1984686X35883

Tabela 3 - Distribuição das publicações conforme a natureza dos estudos

\begin{tabular}{lcc}
\hline NATUREZA & $\mathbf{f}$ & $\%$ \\
\hline Quantitativa & 59 & 79,73 \\
Qualitativa & 5 & 6,76 \\
Quanti-qualitativa & 7 & 9,46 \\
Ensaio teórico & 3 & 4,05 \\
\hline TOTAL & 74 & 100,00 \\
\hline
\end{tabular}

Fonte: Elaboração própria.

Das 74 publicações analisadas, 71 são relatos de pesquisas e três são ensaios teóricos. A partir da Tabela 3, verifica-se que a maioria das pesquisas sobre autoeficácia docente e Educação Especial na perspectiva da educação inclusiva é de natureza quantitativa (59). Tais estudos envolvem a construção e adaptação de instrumentos de medida (SHARMA; LOREMAN; FORLIN, 2012; SCHWAB; HELLMICH; GÖREL, 2017); a avaliação dos níveis de autoeficácia docente em diferentes contextos geográficos, níveis e modalidades de ensino (ALMEIDA et al., 2016; SHARMA; SOKAL, 2015); bem como a correlação entre autoeficácia docente e outros aspectos, por exemplo, as atitudes em relação à inclusão e o burnout (SHARMA; SHAUKAT; FURLONGER, 2015; BOUJUT et al., 2017). Nas cinco pesquisas qualitativas, cujos objetivos estavam voltados para a avaliação da autoeficácia docente em grupos menores, os dados foram coletados por meio de questionários com questões abertas, entrevistas, grupos focais ou relatos escritos (SHANI; HEBEL, 2016; SANINI; BOSA, 2015; LEONARDO, 2017). Foram sete os estudos que integraram os instrumentos quantitativos aos métodos qualitativos e é entre eles que se encontram as três únicas pesquisas interventivas. Uma delas teve a finalidade de ajudar os professores a implementar estratégias pedagógicas inclusivas em sala de aula a partir de discussões em grupo focal (COLEMAN, 2017); enquanto outra, delineou e examinou os efeitos de um modelo de formação sobre a autoeficácia docente (BURTON; PAC, 2009); havendo ainda, a proposição de integração de discussões facilitadas pelo pesquisador e tarefas de autorreflexão a um curso online (BOOMGARD, 2013).

Em relação aos participantes, a maioria das pesquisas abordou professores em serviço, em especial, os que possuem formação generalista, como se observa na Tabela 4: 
Tabela 4 - Distribuição das pesquisas segundo o tipo de atuação/formação dos professores participantes

\begin{tabular}{lcccccc|cc}
\hline \multicolumn{1}{c}{ TIPO DE } & \multicolumn{2}{c}{ EM SERVIÇO } & \multicolumn{2}{c}{ EM FORMAÇÁO } & \multicolumn{2}{c|}{ AMBOS } & \multicolumn{2}{c}{ TOTAL } \\
ATUAÇÃO & $\mathbf{f}$ & $\%$ & $\mathbf{f}$ & $\%$ & $\mathbf{f}$ & $\%$ & $\mathbf{f}$ & $\%$ \\
\hline Generalistas & 30 & 42,25 & 21 & 29,58 & 02 & 2,82 & 53 & 74,65 \\
Especialistas & 07 & 9,86 & 0 & 0 & 0 & 0 & 07 & 9,86 \\
Ambos & 11 & 15,49 & 0 & 0 & 0 & 0 & 11 & 15,49 \\
\hline TOTAL & 48 & 67,60 & 21 & 29,58 & 02 & 2,82 & 71 & 100,00 \\
\hline
\end{tabular}

Fonte: Elaboração própria.

Entre os relatos de pesquisas, 48 estudos tiveram professores em serviço como participantes: sete deles com professores especialistas em Educação Especial, 30, com professores generalistas, e 11, envolvendo ambos os tipos de professores. Outros 21 trabalhos contaram com a participação de professores em formação inicial, e houve ainda, dois estudos que abarcaram tanto professores em atuação quanto em formação.

\section{Pesquisas sobre a formação de professores para o fortalecimento da autoeficácia docente no contexto da educação inclusiva}

Foram 12 os estudos que objetivaram investigar os efeitos da formação sobre a autoeficácia docente para a inclusão do público da Educação Especial, nenhum deles produzido no Brasil. A partir da leitura dos resumos e textos completos, descreveremos brevemente essas pesquisas:

Boomgard (2013) investigou as alterações na autoeficácia e burnout percebidos por professores especialistas e generalistas face às discussões facilitadas pelo pesquisador e tarefas de autorreflexão incorporadas a um curso online sobre autismo, oferecido por uma universidade pública da Califórnia. Os dados indicaram que a autoeficácia dos professores para o trabalho com discentes autistas aumentou significativamente após os cinco fóruns de discussões facilitadas e registros de autorreflexões que ocorriam a cada três semanas e se baseavam nos conteúdos do curso que foi desenvolvido ao longo de 16 semanas.

Forlin, Loreman e Sharma (2014) examinaram as mudanças provocadas por um curso de treinamento para professores de Hong Kong nas atitudes, preocupações e autoeficácia em relação à inclusão. Os resultados revelaram um efeito positivo nos fatores cognitivos investigados, porém, modesto. A média dos professores participantes continuou apontando 
http://dx.doi.org/10.5902/1984686X35883

para uma autoeficácia moderada, visto estar localizada no intervalo de neutralidade da escala.

Almeida et al. (2016) analisaram a percepção de autoeficácia de professores especialistas para o uso do computador no ensino de estudantes com deficiência, antes e depois de participarem de um programa de formação voltado para o desenvolvimento dessa competência. O estudo envolveu professores das zonas urbana e rural e apontou diferenças na autoeficácia desses profissionais segundo a localidade.

Chao et al. (2017) investigaram as mudanças na autoeficácia de professores que participaram de um curso de formação em serviço durante uma semana sobre o ensino de estudantes com necessidades educacionais especiais em Hong Kong. A partir do método teste-reteste, constatou-se um aumento significativo na autoeficácia dos participantes. No entanto, anteriormente ao curso, as médias obtidas na mensuração inicial das três subescalas do instrumento utilizado eram de 6,03; 6,25 e 6,12, aumentando respectivamente para 6,$35 ; 6,50$ e 6,42, quando a pontuação poderia variar entre 1 e 9, o que indica uma autoeficácia moderadamente positiva.

Embora a formação teórica possa favorecer a percepção de autoeficácia para o ensino na diversidade, quando atrelada a vivências práticas, os resultados tendem a ser mais satisfatórios como mostra a pesquisa conduzida por Peebles e Mendaglio (2014) que verificaram as influências de um curso sobre inclusão na formação inicial de professores matriculados em uma universidade do Canadá. O curso tinha duração de 10 semanas, seguidas de outras três em atividades de campo nas escolas da vizinhança. Foram realizados testes em três momentos: no início do curso, ao final na formação teórica e após a experiência de campo, cujas médias da escala utilizada nos diferentes momentos foram respectivamente: 76, 45; 80, 65 e 83, 98, quando a pontuação poderia variar de 18 a 108 . Os dados revelaram que a formação promoveu ganhos significativos em termos de autoeficácia tanto com os estudos teóricos quanto com a experiência prática.

Burton e Pace (2009) delinearam um modelo de formação inicial de professores para o ensino de matemática a alunos com deficiência visando favorecer a autoeficácia e as atitudes dos futuros docentes para a inclusão nos Estados Unidos. Os pesquisadores analisaram os resultados do curso durante três anos, o qual teve 13 participantes em sua primeira edição, oito, na segunda e cinco, na terceira. Inicialmente, o curso era composto por dois módulos teóricos, mas diante das mudanças inexistentes ou mínimas na 
autoeficácia e nas atitudes para inclusão apresentadas pelos cursistas, evidenciadas por testes estatísticos, os pesquisadores acrescentaram um novo módulo à terceira edição. Este módulo envolvia o estudo mais aprofundado de estratégia de ensino, a exibição de um vídeo sobre a inclusão escolar de uma menina com deficiência intelectual e a realização de experiência prática de tutoria em matemática a estudante com deficiência durante 20 horas. Os participantes da última edição registraram suas práticas em um diário, o qual foi analisado qualitativamente, sugerindo que houve aumento na autoeficácia, na confiança e mudança de atitudes para o ensino de matemática a estudantes com deficiência.

Shani e Hebel (2016) objetivaram avaliar as implicações de um programa de treinamento para professores a respeito da inclusão escolar sobre a autoeficácia docente. O curso foi desenvolvido por uma faculdade de Israel e seu diferencial é a integração da experiência prática aos estudos teóricos. Ao final da formação, os participantes revelaram em entrevista, que tal experiência é considerada como o principal promotor do senso de autoeficácia para a inclusão.

Tindall, Culhane e Foley (2016) analisaram os efeitos de um programa de educação física adaptada para o ensino de alunos com deficiência na formação inicial de educadores físicos em uma universidade da Irlanda. Esse programa tinha duração de 10 semanas e, além de palestras, envolvia o desenvolvimento de atividades práticas com duração de uma hora semanal junto a crianças e adultos com deficiência, de maneira que cada estudante ficava responsável por dar atenção e suporte a uma pessoa com deficiência. Essas práticas ocorreram durante quatro semanas. Com base na aplicação de uma escala antes e após a participação no programa, verificou-se uma ampliação significativa na autoeficácia dos futuros professores, o que foi reafirmado em entrevista coletiva ao término da formação. Em contrapartida, há estudos que apontam que a experiência direta nem sempre desencadeia a elevação da autoeficácia.

Lancaster e Bain (2010) conduziram um estudo comparativo entre duas versões de um curso de educação inclusiva para professores de educação pré-escolar em formação inicial. As versões do curso eram ministradas pela mesma universidade australiana, porém, em campi diferentes. Os estudantes do Campus A tiveram 39 horas de aulas na universidade, contendo estudos teóricos, workshops, resolução de problemas, elaboração de planos de aula e colaboração entre pares e feedback; ao passo que os estudantes do Campus $B$ tiveram, além dessas 39 horas, 11 horas de experiência de ensino em programas 
comunitários que atendiam crianças com necessidades especiais no contraturno da escola. Tanto os estudantes do Campus A quanto do Campus B demonstraram um crescimento significativo em termos de autoeficácia. Contudo, não houve diferença estatisticamente significativa entre os resultados dos dois grupos, o que indica que a experiência de campo não afetou a autoeficácia dos estudantes do Campus B.

O estudo de Gao e Mager (2011) analisou a relação entre senso de autoeficácia, crenças sobre diversidade sociocultural e atitudes de professores em formação inicial com os efeitos de um programa sobre inclusão, conduzido por uma universidade privada nos Estados Unidos. Esse programa envolve 522 horas de experiência de campo. Os resultados apontaram para a associação positiva entre autoeficácia, atitudes diante da inclusão e crenças sobre as diferenças socioculturais. Embora os dados apontem para a eficácia do curso no preparo para a inclusão, os participantes não apresentaram alterações nos sentimentos negativos em relação às crianças com deficiências comportamentais.

Woodcock, Hemmings e Kay (2012) analisaram a autoeficácia de estudantes matriculados no terceiro ano de um curso de formação inicial de professores em uma universidade regional da Austrália, antes e após a realização de estudos sobrea inclusão de estudantes da Educação Especial, acompanhados de uma experiência prática. A formação não provocou resultados significativos.

Sharma e Sokal (2015) compararam os efeitos de dois cursos sobre Educação Especial na perspectiva da educação inclusiva nas atitudes, preocupações e autoeficácia docente para a inclusão. Um deles oferecido por uma universidade australiana e outro, por uma universidade canadense, ambos integrantes da formação inicial de professores e diferindo em termos de currículo e práticas de ensino. O curso da universidade canadense tinha duração de 30 horas e acontecia concomitantemente a uma prática em sala de aula inclusiva, porém, alguns dos 60 participantes ainda não tinham realizado a prática devido à parte da coleta ter ocorrido em um período de transição. Na universidade australiana, que contou com 27 participantes, o curso tinha duração de 18 horas e girava em torno de três questões: O que é inclusão? Por que incluir? Como incluir a todos?. Adicionalmente, há uma breve descrição de um estudo de caso. Com base na aplicação de escalas antes e após os cursos, obtiveram-se resultados positivos e significativos em relação à autoeficácia dos estudantes das duas universidades, bem como também foi verificada a diminuição das preocupações. No entanto, as atitudes para a inclusão melhoraram somente no grupo 
australiano, havendo um decrescimento estatisticamente significativo entre os estudantes canadenses, justamente os que possuíam prática integrada. Destaca-se que os resultados australianos foram mais favoráveis nos três quesitos analisados. Nessa perspectiva, os autores chamam a atenção para os riscos da prática de estágio junto a professores cujas práticas pedagógicas não são eficazes.

As pesquisas sobre autoeficácia docente para a inclusão do público da Educação Especial nos mostram que apesar de ser possível o favorecimento das crenças dos professores em suas capacidades a partir de conhecimentos teóricos, as vivências práticas aumentam a probabilidade de obtenção de bons resultados. A experiência de domínio é apontada como a fonte de autoeficácia mais potente (BANDURA, 1997). Contudo, quando a prática não é bem-sucedida, pode ocasionar o enfraquecimento das crenças de autoeficácia. Por esse prisma, salientamos as potencialidades das experiências vicárias, isto é, aquelas que provêm da observação de outras pessoas atuando, de maneira a propiciar a comparação com os sucessos do outro e a aquisição de competências. Ao visualizar que os esforços de outros desencadeiam em êxito, o observador tem uma ampliação das crenças sobre suas possibilidades de obter sucesso em atividades semelhantes, do mesmo modo em que ao observar que os esforços produzidos resultam em fracasso, há um decréscimo de sua autoeficácia e, consequentemente, de sua motivação. Quanto maior é a similaridade entre o observador e o observado, diretamente proporcional é o efeito persuasivo desencadeado (ANDREU; PALLARÉS; JUÁREZ, 2004). Logo, convém expor os professores a situações educacionais inclusivas bem-sucedidas, o que pode ocorrer por meio da tecnologia de vídeo. As experiências vicárias são uma importante fonte de autoeficácia docente e se mostram pouco exploradas cientificamente, sobretudo, no contexto da Educação Especial.

\section{Considerações finais}

A autoeficácia docente está relacionada ao esforço e à motivação dos professores e afeta o desempenho de suas atividades laborais, o que também é válido para a efetivação da inclusão escolar, com destaque para os discentes com deficiência, transtornos globais ou altas habilidades/superdotação.

São poucas as pesquisas brasileiras que se dedicaram a investigar a autoeficácia docente e a Educação Especial na perspectiva da educação inclusiva e, nenhuma delas abordou a formação docente. No contexto internacional, observa-se que diversas 
pesquisas tiveram como objeto de estudo a autoeficácia docente em relação à Educação Especial, porém, as que analisaram os efeitos da formação no fortalecimento da autoeficácia para práticas inclusivas não atingiram um número expressivo. A análise destas pesquisas revela que a formação pode aumentar os níveis de autoeficácia docente e que tal probabilidade é ampliada na medida em que há articulação entre teoria e prática. No entanto, quando a prática não resulta em sucesso, pode abalar a autoeficácia, portanto, ressaltamos as potencialidades das experiências vicárias na formação de professores para práticas inclusivas, seja na formação inicial ou continuada, a fim de que a observação de situações educacionais inclusivas bem-sucedidas propicie a aquisição de novas habilidades e o fortalecimento da autoeficácia docente.

\section{Referências}

ALMEIDA, Conceicao M. et al. Urban and Rural Preservice Special Education Teachers' Computer Use and Perceptions of Self-Efficacy. Rural Special Education Quarterly, v. 35, n. 03, p. 12-19, set. 2016.

ANDREU, Llorenç; PALLARÉS, Àlex; JUÁREZ, Óscar. La incidência del entrenamiento en el nível de eficácia percebida: un estudio en un equipo feminino de fútbol. In: SALANOVA, Marisa [et al.]. Nuevos horizontes em la investigación sobre la autoeficacia. Castelló de la Plana: Publicacions de la Universitat Jaume I, D.L. 2004, p. 205-211.

AZZI, Roberta Gurgel; POLYDORO, Soely Aparecida Jorge; BZUNECK, José Aloyseo. Considerações sobre a auto-eficácia docente. In: AZZI, Roberta Gurgel; POLYDORO, Soely Aparecida Jorge (Orgs.). Auto-eficácia em diferentes contextos. Campinas, SP: Editora Alínea, 2006, p. 149-159.

BANDURA, Albert. Regulation of Cognitive Process Through Perceived Selfefficacy. Developmental Psychologycal, v. 25, n. 05, p. 729-735, 1989.

BANDURA, Albert. Self-efficacy: the exercise of control. New York: W.H. Freeman and Company, 1997.

BOOMGARD, Monica. Changes in perceived teacher self-efficacy and burnout as a result of facilitated discussion and self-reflection in an online course designed to prepare teachers to work with students with autism. San Francisco: USF, 2013. 212f. Tese (Doutorado em Educação), Faculdade da Escola de Educação, Universidade de San Francisco, 2013.

BOUJUT, Emilie et al. Self-efficacy and burnout in teachers of students with autism spectrum disorder. Research in Autism Spectrum Disorders, v. 36, p. 08-20, Abr. 2017. 
BRASIL. LDB - Lei de Diretrizes e Bases da Educação Nacional: Lei oㅡ 9394, de 20 de dezembro de 1996, que estabelece as diretrizes e bases da educação nacional. Brasília: Senado Federal, Coordenação de Edições Técnicas, 1996.

BURTON, Dolores; PACE, Darra. Preparing Pre-Service Teachers to Teach Mathematics in Inclusive Classrooms: A Three-Year Case Study. School Science and Mathematics, v. 109, n. 02, p. 108-115, Fev. 2009.

CAPES. Resultado da Avaliação Quadrienal 2017. Disponível em: http://avaliacaoquadrienal.capes.gov.br/resultado-da-avaliacao-quadrienal-20172. Acesso em: 31 de julho de 2018.

CHAO, Chih Nuo Grace et al. Improving teachers' self-efficacy in applying teaching and learning strategies and classroom management to studentes with special education needs in Hong Kong. Teaching and Teacher Education, v. 66, p. 360-369, ago. 2017.

COLEMAN, Michael E. Enhancing teacher efficacy and pedagogical practices amongst general and special education teachers. Estados Unidos: Rowan University, 2017. 242f. Tese (Doutorado em Educação), Faculdade de Educação, Rowan University, 2017.

FORLIN, Chris; LOREMAN, Tim; SHARMA, Umesh. A system-wide professional learning approach about inclusion for teachers in Hong Kong. Asia-Pacific Journal of Teacher Education, v. 42, n. 03, p. 247-260, abr. 2014.

GAO, Wei; MAGER, Gerald. Enhancing Preservice Teachers' Sense of Efficacy and Attitudes toward School Diversity through Preparation: A Case of One U.S. Inclusive Teacher Education Program. International Journal of Special Education, v. 26, n. 02, p. 92-107, 2011.

IAOCHITE, Roberto Tadeu et al. Autoeficácia no campo educacional: revisão das publicações em periódicos brasileiros. Psicologia Escolar e Educacional, v. 20, n. 01, p. 45-54, jan./abr. 2016.

LANCASTER, Julie; BAIN, Alan. The design of pre-service inclusive education courses and their effects on self-efficacy: A comparative study. Asia-Pacific Journal of Teacher Education, v. 38, n. 02, p.117-128, 2010.

LEONARDO, Fátima Cristina Luiz. Associações entre crenças e autoeficácia e estratégias inclusivas adotadas por professores universitários. Presidente Prudente: UNOESTE, 2017. 68f. Dissertação (Mestrado em Educação), Universidade do Oeste Paulista, Presidente Prudente, 2017.

MARTÍNEZ, Isabel M. Autoeficacia aplicada al trabajo y a las organizaciones. In: SALANOVA, Marisa [et al.]. Nuevos horizontes en la investigación sobre la autoeficacia. Castelló de la Plana: Publicacions de la Universitat Jaume I, D.L. 2004, p. $178-186$.

OMOTE, Sadao. Produção Acadêmica em Educação Especial. In: OMOTE, Sadao; OLIVEIRA, Anna Augusta Sampaio; CHACON, Miguel Claudio Moriel. Ciência e 
Conhecimento em Educação Especial. São Carlos: Marquezine \& Manzini: ABPEE, 2014, p. 13-24.

PEEBLES, Jodi L.; MENDAGLIO, Sal. The impact of direct experience on preservice teachers' self-efficacy for teaching in inclusive classrooms. International Journal of Inclusive Education, v. 18, n. 12, p. 1321-1336, abr. 2014.

SANINI, Cláudia; BOSA, Cleonice Alves. Autismo e inclusão na educação infantil: Crenças e autoeficácia da educadora. Estudos psicológicos, Natal, v. 20, n. 03, p.173-183, jul./set. 2015.

SCHMID, Mônica Bossa dos Santos. Autoeficácia de professores: análise de modelo de intervenção para o uso das tecnologias digitais da informação e da comunicação. 2015. 142f. Dissertação (Mestrado em Educação) - Centro de Educação, Comunicação e Artes, Universidade Estadual de Londrina, Londrina, 2015.

SCHWAB, Susanne; HELLMICH, Frank; GÖREL, Gamze. Self-Efficacy of Prospective Austrian and German Primary School Teachers Regarding the Implementation of Inclusive Education. Journal of Research in Special Educational Needs, v. 17, n. 03, p. 205-217, fev. 2017.

SHANI, Michal; HEBEL, Orly. Educating towards Inclusive Education: Assessing a Teacher-Training Program for Working with Pupils with Special Educational Needs and Disabilities (SEND) Enrolled in General Education Schools. International Journal of Special Education, v. 31, n. 03, 2016.

SHARMA, Umesh; LOREMAN, Tim; FORLIN, Chris. Measuring teacher efficacy to implement inclusive practices. Journal of Research in Special Educational Needs, v. 12, n. 01, p. 12-21, 2012.

SHARMA, Umesh; SHAUKAT, Sadia; FURLONGER, Brett. Attitudes and Self-Efficacy of Pre-Service Teachers towards Inclusion in Pakistan. Journal of Research in Special Educational Needs, v.15, n. 02, p. 97-105, abr. 2015.

SHARMA, Umesh; SOKAL, Laura. The Impact of a Teacher Education Course on PreService Teachers' Beliefs about Inclusion: An International Comparison. Journal of Research in Special Educational Needs, v. 15, n. 04, p. 276-284, out. 2015.

SILVA, Régis Henrique dos Reis; GAMBOA, Sílvio Sánchez. Análise epistemológica da pesquisa em educação especial: a construção de um instrumental de análise. Atos de pesquisa em Educação - PPGE/ME FURB, v. 06, n. 02, p. 373-402, mai./ago. 2011.

TINDALL, Daniel; CULHANE, Maeve; FOLEY, John. Pre-service teachers' selfefficacy towards children with disabilities: An Irish perspective. European Journal of Adapted Physical Activity, v. 09, n. 01, p. 27-40, 2016.

VENDITTI JÚNIOR, Rubens. Análise da auto-eficácia docente de professores de educação física. Campinas: UNICAMP, 2005. 167f. Dissertação (Mestrado em Educação Física) - Faculdade de Educação Física, Universidade Estadual de Campinas, Campinas, 2005. 
VENDITTI JÚNIOR, Rubens. Auto-eficácia docente e motivação para a realização do(a) professor(a) de educação física adaptada. Campinas: UNICAMP, 2010. 315f. Tese (Doutorado em Educação Física) - Faculdade de Educação Física, Universidade Estadual de Campinas, Campinas, 2010.

WOODCOCK, Stuart, HEMMINGS, Brian, KAY, Russell. Does study of an inclusive education subject influence pre-service teachers' concerns and self-efficacy about inclusion? Australian Journal of Teacher Education, v. 37,n. 06, p. 1-11, jun. 2012.

\section{Correspondência}

Bárbara Amaral Martins - Universidade Federal do Mato Grosso do Sul, Campus do Pantanal, Av. Rio Branco, 1270 - Universitário, Corumbá - MS, CEP 79304-902.

\section{(C) $(1) \Theta$}

This work is licensed under a Creative Commons Attribution-NonCommercial 4.0 International (CC BY-NC 4.0) 